\title{
Focused and collimated Gaussian laser beam scintillation in weakly turbulent marine atmospheric medium
}

\author{
Ahmed A. Abbas ${ }^{1}$, Hamza Gerçekcioğlu² ${ }^{2}$ H. Haldun Göktaş ${ }^{3}$ \\ ${ }^{1}$ College of Engineering, University of Anbar, Iraq \\ ${ }^{2}$ Ministry of Transport, Maritime Affairs and Communications, Turkey \\ ${ }^{3}$ College of Engineering, Ankara Yıldırım Beyazıt University, Turkey
}

\begin{tabular}{l} 
Article Info \\
\hline Article history: \\
Received Dec 10, 2019 \\
Revised Jun 10, 2020 \\
Accepted Jun 28, 2020 \\
\hline
\end{tabular}

\section{Keywords:}

Atmospheric propagation Atmospheric turbulence Free-space

Optical communication

Scintillation

\begin{abstract}
The scintillation index on the axis for Gaussian beams focused and collimated in weak marine turbulence is formulated via the usage of Rytov method. The average bit error rate $\langle\mathrm{BER}\rangle$ is evaluated using this formulation. The scintillation index and $\langle\mathrm{BER}\rangle$ versus propagation distance and source size are determined by using the log-normal distributed. Intensity for the collimated and focused. Gaussian beams, which are exhibited for wavelength, source size, focal length, and $\langle\mathrm{SNR}\rangle$. The focused beams are revealing more advantageous than collimated beams in an atmospheric marine environment. The findings of this study are significant for optical communication system performance in this layer.
\end{abstract}

This is an open access article under the CC BY-SA license.

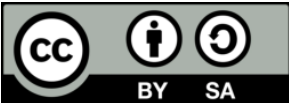

\section{Corresponding Author:}

Ahmed A. Abbas,

College of Engineering,

The University of Anbar,

Anbar, Iraq.

Email: ahmed.abbas@uoanbar.edu.iq

\section{INTRODUCTION}

The atmospheric marine environment is one that is in immediate contact with water and directly impacted by seas and oceans. In this media, the ocean and atmosphere alternate between tremendous amounts of moisture, heat, and momentum particularly by turbulent transport. Thus, studying turbulence in the marine environment is important to ensure efficient optical communication systems and in the design of nautical laser radar. Atmospheric turbulence is considered one of the extremely important factors for efficiency-limiting of the communication links in free-space optical (FSO). Scintillation is a vital consequence of the turbulence, which generates depth inconstancy within the signal received and consequently leads to link unreliability. In this study, the focus is scintillation in the marine environment. The findings on the scintillation index of focused and collimated Gaussian laser beams propagating in this environment are all reported $[1,2]$.

Numerous studies have been conducted on various effects of distinct turbulence on the marine atmosphere. In this paper, only the important positive expressions are reported. In the marine atmosphere, the power spectra are stated in [3,4]. Utilizing on-axis scintillation from higher order laser beams in a weak turbulent marine environment medium were assessed and presented in [5, 6]. Starting a generalized maritime atmospheric spectrum model to present Gaussian beam waves propagating through non-Kolmogorov maritime atmospheric environment where an analytic expression is derived of average scintillation index which is associated with the receiver-aperture [7]. The marine atmospheric turbulence modulation transfer 
function characterizes the results of marine atmospheric turbulence on a system with optical imaging [8]. The spread and wander beams of a Gaussian beam wave were anatomized by propagating through marine atmospheric turbulence [9]. The beam wave front correction in maritime atmosphere was studied by using adaptive optics [10, 11]. Using random air-water interface through an adaptive optics system caused mitigating signal distortions therefore this new concept described in [12]. In the maritime environment, field measurements are used to offer most important contribution specified by the type of methodology to compute the probability density function of the laser beam intensity [13].

The angle of arrival fluctuations in the maritime atmospheric turbulence is examined [14, 15]. Among key interim data is irradiance scintillation's temporal power spectra over weakened marine disturbance which is examined analytically and numerically in each element [16]. In addition, such to key interim data is to depict the correlation of irradiance inconstancy at unique time situations [17]. In this study, we derived and evaluated the. Scintillation index through weakened atmospheric marine turbulence by using Rytov method. Our goal is to comprehend how the use of focused and collimated Gaussian beams helps in shrinking the on-axis scintillation in wireless optical communication in horizontal links in this layer.

\section{FORMULATION}

This section is derived for the scintillation index on the axis for Gaussian beams focused and collimated in weak marine turbulence. The power spectrum of marine refractive index fluctuation in spatial domain is as follows $[1,2]$ :

$$
\Phi_{n}(\kappa)=0.033 C_{n}^{2} k^{2}\left[1-0.061 \kappa / \kappa_{H}+2.836 \kappa^{7 / 6} / \kappa_{H}{ }^{7 / 6}\right] \frac{\exp \left(-\kappa^{2} / \kappa_{H}^{2}\right)}{\left(\kappa_{0}^{2}+\kappa^{2}\right)^{11 / 6}},
$$

where $\kappa_{H}=\frac{3.14}{l_{0}}, \kappa_{0}=\frac{1}{L_{0}}$, the scales of turbulence outer and inner $L_{0}$ and $l_{0}, \kappa$ refers to the wave number (spatial) for $0 \leq \kappa<\infty$. The scintillation index based on Rytov approximation for homogeneous and isotropic turbulence is presented as [17].

$$
m^{2}=4 \pi R e\left\{\int_{0}^{L} d \eta \int_{0}^{\infty} \kappa d \kappa \int_{0}^{2 \pi} d \theta[q m 1(\eta, \kappa, \theta)+q m 2(\eta, \kappa, \theta)] \Phi_{n}(\kappa)\right\},
$$

In which $(\kappa, \theta)$ indicate to the polar coordinate illustration of the two-dimensional spatial frequency, $\eta$ denoting a length along the propagation axis which is a variable, $\mathrm{L}$ is the propagation distance of the link and $\operatorname{Re}(*)$ denotes the real part of the argument [17]. The expressions in the integrand of (2) are

$$
\begin{aligned}
& q m 1(\eta, \kappa, \theta)=\frac{-k^{2}}{Q^{2}(L)} \frac{1}{(1+i \alpha L)^{2}} \exp \left[\frac{-i(L-\eta)(1+i \alpha \eta) \kappa^{2}}{k(1+i \alpha L)}\right] \\
& q m 2(\eta, \kappa, \theta)=\frac{k^{2}}{|Q(L)|^{2}} \frac{1}{(1+i \alpha L)\left(1-i \alpha^{*} L\right)} \exp \left[\frac{-i(L-\eta)}{2 k}\left(\frac{1+i \alpha \eta}{1+i \alpha L}-\frac{1-i \alpha^{*} \eta}{1-i \alpha^{*} L}\right) \kappa^{2}\right], \\
& Q(L)=1 /(1+i \alpha L),
\end{aligned}
$$

where "*" is the complex. Conjugate, $i=(-1)^{0.5,} k=2 \pi / \lambda$ is a wavenumber, $\alpha=\frac{1}{k\left(\alpha_{S}\right)^{2}}+\frac{i}{F_{S}}$ here $\alpha_{s}$ and $F_{s}$ are the source size and focal length of the Gaussian beam and incident field expression is $\exp \left(-1 / 2 k \alpha s^{2}\right)[18,19]$. Inserting (3), (4) and the power spectrum of marine in (2), acting the integration over $\kappa$ from [20]. Then, the scintillation index on the axis for Gaussian beams focused and collimated. In weakened marine turbulence is found and demonstrated as:

$$
\begin{aligned}
& m^{2}=1.39 k^{2} C_{n}^{2} \operatorname{Re}\left\{\int _ { 0 } ^ { L } d \eta \sum _ { n = 0 } ^ { \infty } \frac { 1 } { n ! } \left[\left[\left(\kappa_{0}^{2}\right)^{n-\frac{5}{6}} \Gamma\left(-n+\frac{5}{6}\right) \Gamma(n+1)\right]\right.\right. \\
& \times\left[\left(\frac{-1}{Q^{2}(L)(1+i \alpha L)^{2}}\right)\left[-\left(\frac{i(L-\eta)(1+i \alpha \eta)}{k(1+i \alpha L)}+\frac{1}{\kappa_{H}^{2}}\right)\right]^{n}+\left(\frac{1}{|Q(L)|^{2}(1+i \alpha L)\left(1-i \alpha^{*} L\right)}\right)\right. \\
& \left.\times\left[-\left(\frac{i(L-\eta)}{2 k}\left(\frac{1+i \alpha \eta}{1+i \alpha L}-\frac{1-i \alpha^{*} \eta}{1-i \alpha^{*} L}\right)+\frac{1}{\kappa_{H}^{2}}\right)\right]^{n}\right]-\frac{0.061}{\kappa_{H}}\left[\left(\kappa_{0}^{2}\right)^{n-\frac{1}{3}} \Gamma\left(-n+\frac{1}{3}\right)\right. \\
& \left.\times \Gamma\left(n+\frac{3}{2}\right)\right]\left[\left(\frac{-1}{Q^{2}(L)(1+i \alpha L)^{2}}\right)\left[-\left(\frac{i(L-\eta)(1+i \alpha \eta)}{k(1+i \alpha L)}+\frac{1}{\kappa_{H}^{2}}\right)\right]^{n}\right. \\
& \left.+\left(\frac{1}{|Q(L)|^{2}(1+i \alpha L)\left(1-i \alpha^{*} L\right)}\right)\left[-\left(\frac{i(L-\eta)}{2 k}\left(\frac{1+i \alpha \eta}{1+i \alpha L}-\frac{1-i \alpha^{*} \eta}{1-i \alpha^{*} L}\right)+\frac{1}{\kappa_{H}^{2}}\right)\right]^{n}\right]
\end{aligned}
$$




$$
\begin{aligned}
& +\frac{2.836}{\frac{7}{\kappa_{H}^{6}}}\left[\left(\kappa_{0}^{2}\right)^{n-\frac{1}{4}} \Gamma\left(-n+\frac{1}{4}\right) \Gamma\left(n+\frac{19}{12}\right)\right]\left[\left(\frac{-1}{Q^{2}(L)(1+i \alpha L)^{2}}\right)\right. \\
& \times\left[-\left(\frac{i(L-\eta)(1+i \alpha \eta)}{k(1+i \alpha L)}+\frac{1}{\kappa_{H}^{2}}\right)\right]^{n} \\
& \left.\left.\left.+\left(\frac{1}{|Q(L)|^{2}(1+i \alpha L)\left(1-i \alpha^{*} L\right)}\right)\left[-\left(\frac{i(L-\eta)}{2 k}\left(\frac{1+i \alpha \eta}{1+i \alpha L}-\frac{1-i \alpha^{*} \eta}{1-i \alpha^{*} L}\right)+\frac{1}{\kappa_{H}^{2}}\right)\right]^{n}\right]\right]\right\} .
\end{aligned}
$$

Lessen (6) to scintillation index in Kolmogorov to affirm the accuracy of our model and by way of taking inner scale $l_{0}=0$. In addition, neglect the influence of outer scale [1]. The scintillation index in Kolmogorov in [21] matched with our model after reducing. BER and SNR are utilized to determine the quality of the communication systems. BER performance depends on the scintillation strength, receiver noise, and average received power. In weakened marine turbulence, average bit error rate $\langle B E R\rangle$ is presented as follows [22, 23].

$$
<B E R>=0.5 \int_{0}^{\infty} P_{I}(u) \operatorname{erfc}\left(\frac{0.5<S N R>u}{\sqrt{2}}\right) d u,
$$

where $\langle S N R\rangle$ is average signal to noise ratio, $u$ represents unity normalized. Signal, $\operatorname{erfc}($.$) is$ the function of complementary error, and finally $P_{I}(u)$ is expressed in the mathematical $(8)[24,25]$ :

$$
P_{I}=\frac{1}{u m \sqrt{2 \pi}} \exp \left\{-\frac{\left[\ln (u)+0.5 m^{2}\right]^{2}}{2 m^{2}}\right\}, \quad u>0,
$$

\section{RESULTS}

This section presents the numerical results for on-axis scintillation index for collimated Gaussian beams and focused Gaussian beams in marine atmosphere versus the changes in the source size, link length, focal length, and wavelength. Additionally, <BER> versus source size, link length, and wave length are also presented and discussed. All these results are acquired from (6) and (7). Most results are obtained by regulating the wavelength $\lambda=1550 \mathrm{~nm}$, inner scale $=2 \mathrm{~mm}$, outer scale $L_{0}=1 \mathrm{~m}$ and structure constant $C_{n}^{2}=1 \times 10^{-15} \mathrm{~m}^{-2 / 3}$. We prefer $\lambda=1550 \mathrm{~nm}$ because these lasers are most broadly used commercially because they are safe for the eyes and the intensity is attenuated in FSO links.

In Figure 1, the length links versus sintillation index with three source-size values 1,2 , and $3 \mathrm{~cm}$ when the focal length is $F_{S}=10^{7} \mathrm{~m}$ (collimated Gaussian beams) and $F_{s}=L$ (focused Gaussian beams). The collimated Gaussian beams have approximately the same scintillations in comparison with focused Gaussian beams at $\alpha_{s}=1 \mathrm{~cm}$ for all propagation distances. The lowest scintillation are exhibited at all propagation for the focused Gaussian beam At $\alpha_{s}=2$, and $3 \mathrm{~cm}$.

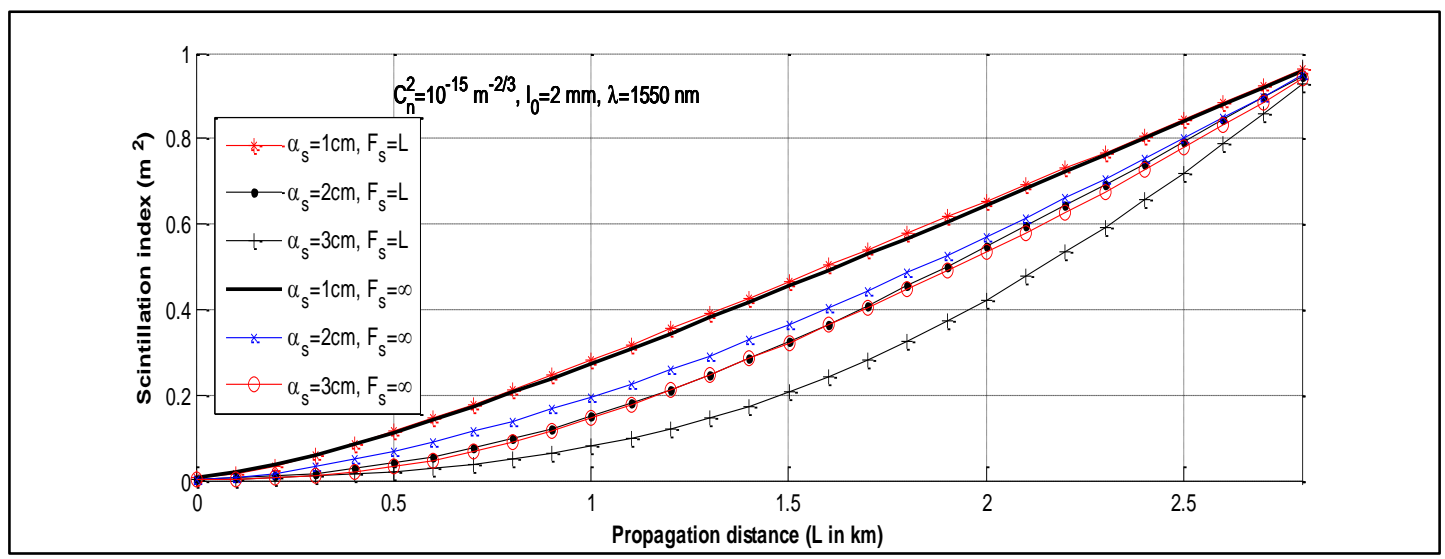

Figure 1. The scintillation index against the propagation distance for different source sizes $\alpha_{s}=1,2,3 \mathrm{~cm}$

With three wavelengths 780,1064 , and $1550 \mathrm{~nm}$, the scintillation index versus the length links are indicated in Figure 2. The figure appears that scintillation index concern on the wavelength and will increase with distance. The variance in atmospheric marine turbulence increases because the transmission range 
increases. Increased wavelength can shrink the scintillation index in weak marine turbulence for both collimated and focused Gaussian beam, but scintillation becomes lower with a focused Gaussian beam.

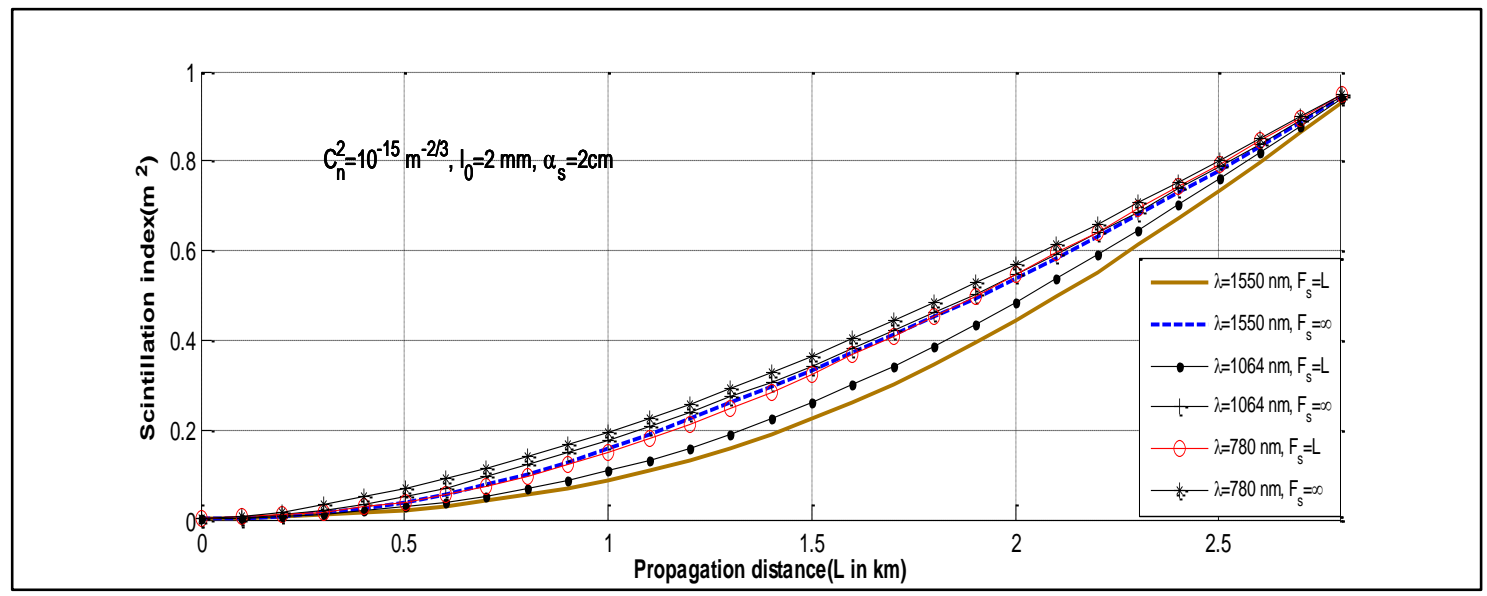

Figure 2. The scintillation index against the propagation distance for collimated and concentrated gaussian beam with different wavelengths $\lambda=0.78,1.064,1.55 \mu \mathrm{m}$

For focused Gaussian beams, Figure 3 exhibits the scintillation index versus source size with $\mathrm{L}=0.5,1,1.5$, and $2.5 \mathrm{~km}$. The figure indicates the highest source size produce low scintillation index with all $\mathrm{L}$. The scintillation versus $\mathrm{L}$ is shown in Figure 4 , with different focal lengths $F_{S}=L$ (focused beam), $10^{4}, 10^{5}$, and $10^{7} \mathrm{~m}$ (collimated beam). From which the concentrated Gaussian beams will return the best scintillation index results in weakened marine turbulence. In Figure 5, $\log (\langle\mathrm{BER}\rangle)$ is depicted versus the source size for the focused Gaussian beam at propagation distances $\mathrm{L}=0.5,1,1.5$, and $2.5 \mathrm{~km}$ and $\langle$ SNR $>=15 \mathrm{~dB}$. Particularly at reasonably sizable source sizes, the <BER $>$ turns into extremely smaller when the concentrations of Gaussian beam is at the propagation Distance $\mathrm{L}=0.5 \mathrm{~km}$. Figure 6 exhibited $\log$ ( $\langle\mathrm{BER}\rangle$ ) versus $\mathrm{L}$ with $\lambda=780,1064$, and $1550 \mathrm{~nm}$. As $\mathrm{L}$ increases, so $<$ BER> raises too. For three values of wavelengths, a marked improvement is observed in the performance in free space links at higher wavelengths for collimated and focused Gaussian beam, but the best improvement accrues with the focused Gaussian beams.

Figure 7 illustrates the performance of $\log \langle\mathrm{BER}\rangle$ as a distance function with $\langle\mathrm{SNR}\rangle=5$ and $15 \mathrm{~dB}$ and $\alpha_{s}=1$ and $2 \mathrm{~cm}$. In Figure 7 the $<\mathrm{BER}>$ increases as $\mathrm{L}$ increases and improves in links of atmospheric marine are related to larger $\langle\mathrm{SNR}\rangle$ and source size for focused Gaussian beams.

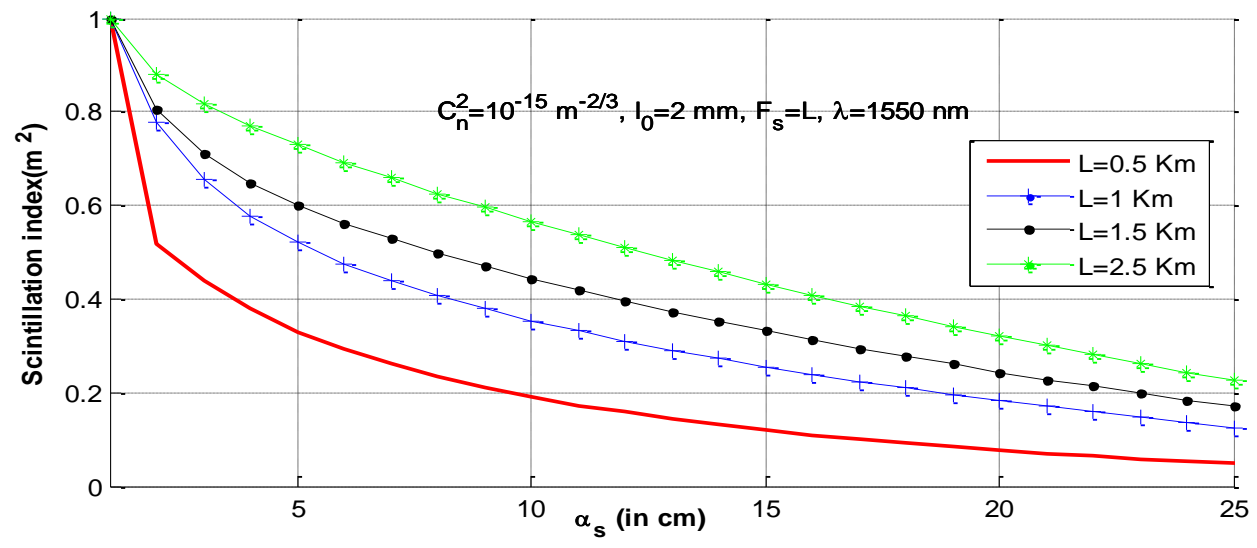

Figure 3. The scintillation index against the source size for various distances of propagation $\mathrm{L}=0.5,1,1.5$, and $2.5 \mathrm{~km}$ and $F_{S}=L$ 


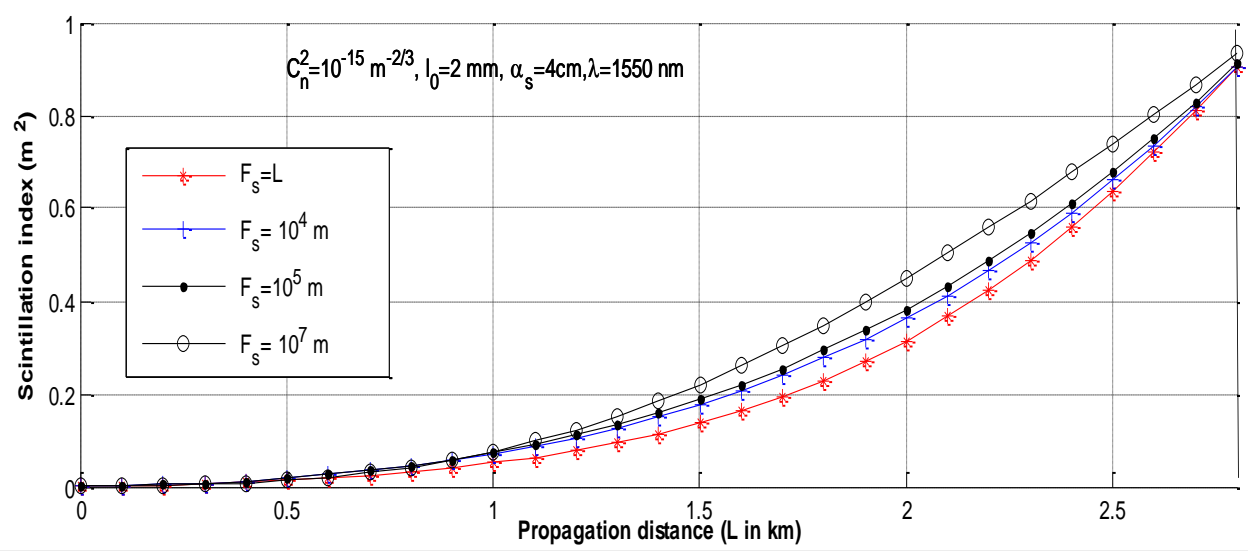

Figure 4. The scintillation index against the propagation distance for different focal lengths $F_{S}=L$ (focused beam), $10^{4}, 10^{5}$, and $10^{7} \mathrm{~m}$

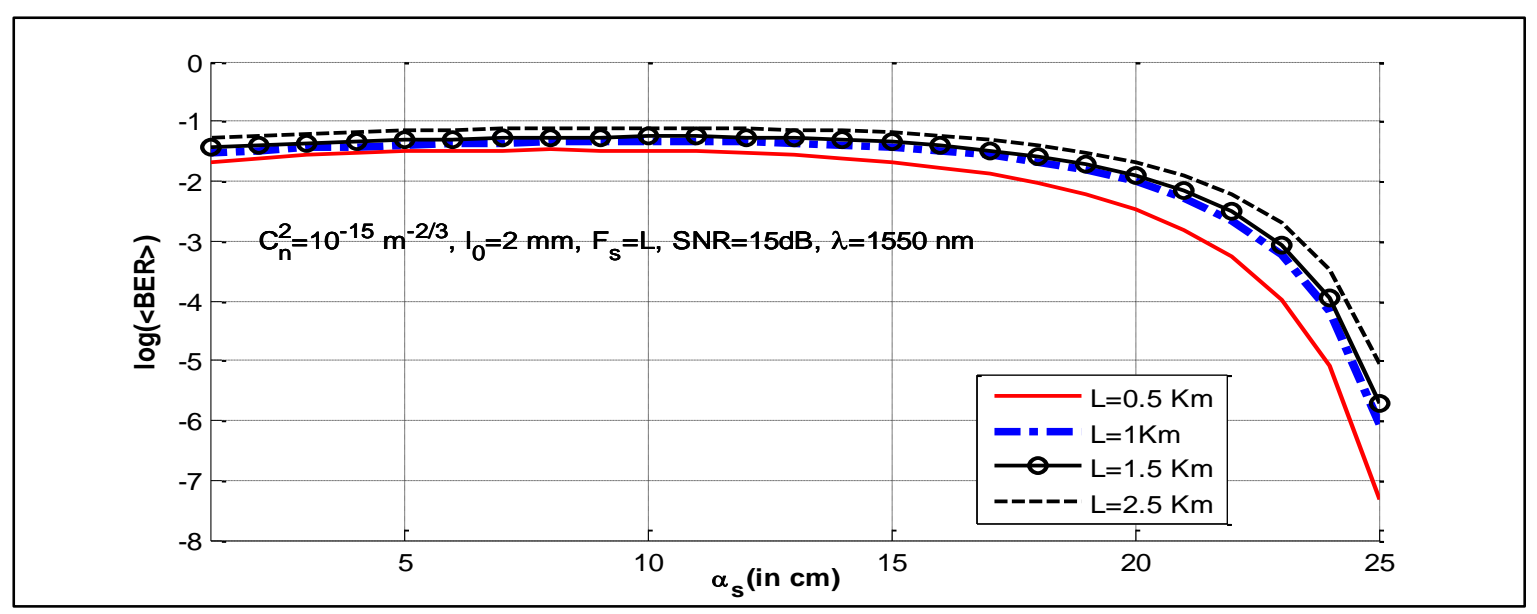

Figure 5. $\langle\mathrm{BER}>$ against source size for different propagation distances $\mathrm{L}=0.5,1,1.5$, and $2.5 \mathrm{~km}$, $\langle\mathrm{SNR}\rangle=15 \mathrm{~dB}$ and $F_{S}=L$

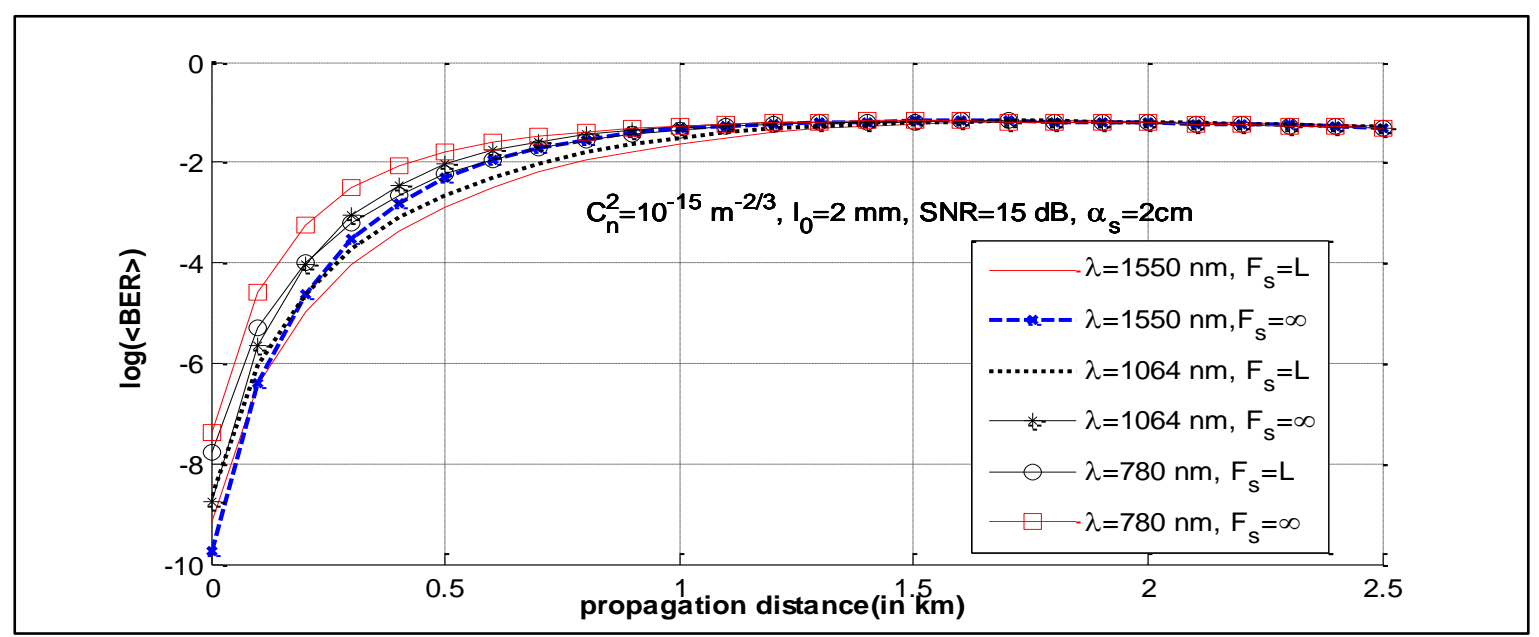

Figure 6. $\angle \mathrm{BER}>$ against propagation distance for different wavelengths $\lambda=0.78,1.064,1.55 \mu \mathrm{m}$, $\langle$ SNR $>=15 \mathrm{~dB}$ 


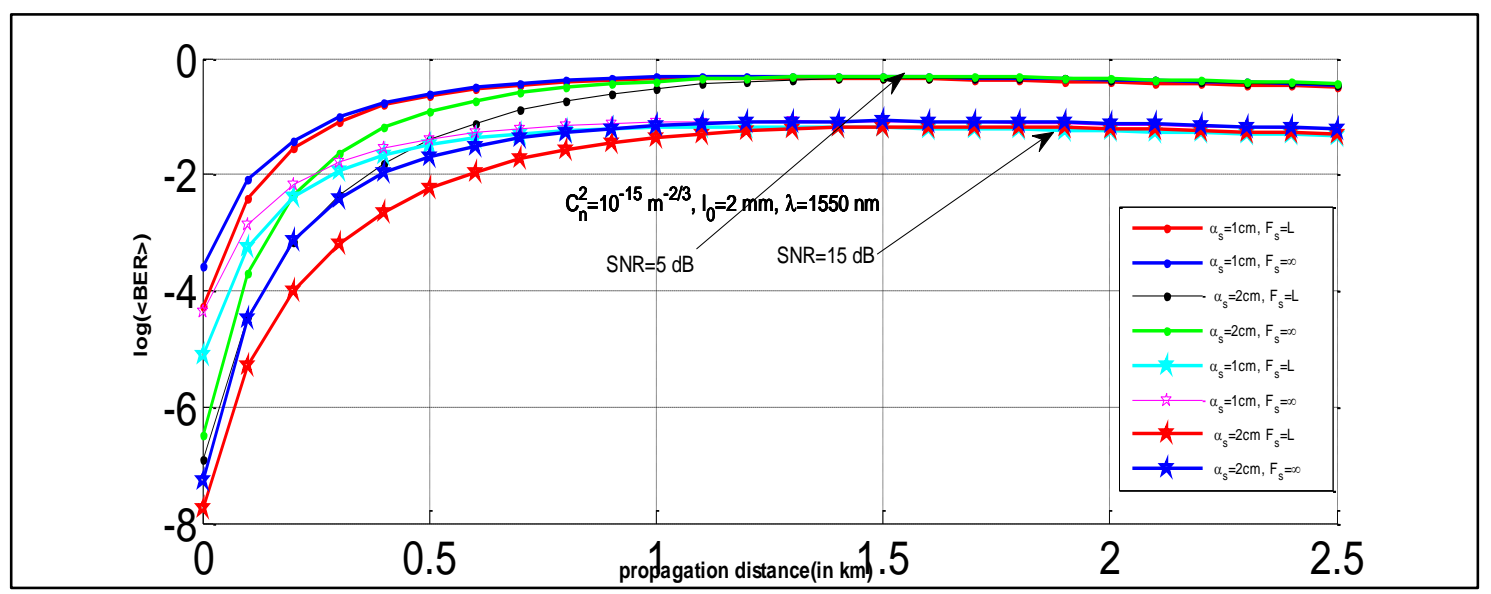

Figure 7. $\langle\mathrm{BER}\rangle$ against propagation distance for different $\langle\mathrm{SNR}\rangle=5$ and $15 \mathrm{~dB}$, and source sizes $\alpha_{s}=1$, and $2 \mathrm{~cm}$

\section{CONCLUSION}

The formulation and evaluation of the scintillation for Gaussian beams focused and collimated in weak marine turbulence is for the horizontal links via the usage of Rytov method. The obtained results is for scintillation index and $\langle\mathrm{BER}\rangle$ versus the link length for beam size, wavelength, focal length, and $\langle\mathrm{SNR}\rangle$. However, the results show that, lowest scintillations at all propagation distances whenever increasing the wavelength and source size of the focused Gaussian beam in weak atmospheric turbulence. Furthermore, the atmospheric marine in horizontal links, <BER> becomes smaller in the case of short length links, and when the wavelength and source size increase. We noticed that the scintillation index has been reduced using the focused beams and therefore the average BER consequently has been improved. The findings can be a beneficial reference in performance and design evaluations of the horizontal links of optical wireless communication that are through marine media, i.e., above seas and oceans. Here, the scintillation index was found just for Gaussian excitation, it can be found for other excitations like cos-Gaussian, cosh-Gaussian, sine-Gaussian, sinh-Gaussian, Hermite-cosh-Gaussian, Hermite-sine-Gaussian, Hermite-sinh-Gaussian; Hermite-cos-Gaussian beams....etc. These excitations may reduce the effect of scintillation and improve in optical links.

\section{REFERENCES}

[1] F. S. Vetelino, et al., "A new marine atmospheric spectrum for laser propagation," Proceedings of SPIE, Atmospheric Propagation IV, vol. 6551, 2007.

[2] K. J. Grayshan, et al., "A marine atmospheric spectrum for laser propagation," Waves in Random and Complex Media, vol. 18, no. 1, pp. 173-184, 2008.

[3] C. A. Friehe, et al., "Effects of temperature and humidity fluctuations on the optical refractive index in the marine boundary layer," Journal of the Optical Society of America A, vol. 65, no. 12, pp. 1502-1511, 1975.

[4] L. C. Andrews, "An analytical model for the refractive index power spectrum and its application to optical scintillations in the atmosphere," Journal of Modern Optics, vol. 39, no. 9, pp. 1849-1853, 1992.

[5] Y. Baykal, "Higher-order laser beam scintillation in weakly turbulent marine atmospheric medium," Journal of the Optical Society of America, vol. 33, no. 4, pp. 758-763, 2016.

[6] M. Q. Taha, et al., "Two-level scheduling scheme for integrated 4G-WLAN network," International Journal of Electrical and Computer Engineering (IJECE), vol. 10, no. 3, pp. 2633-2643, 2020.

[7] M. Cheng, et al., "Scintillation and aperture averaging for Gaussian beams through non-Kolmogorov maritime atmospheric turbulence channels," Optics Express, vol. 23, no. 25, pp. 32606-32621, 2015.

[8] L. Cui, et al., "Atmospheric turbulence MTF for infrared optical waves' propagation through marine atmospheric turbulence," Infrared Physics \& Technology, vol. 65, pp. 24-29, 2014.

[9] I. Toselli, et al., "Gaussian beam propagation in maritime atmospheric turbulence: long term beam spread and beam wander analysis," Proceedings of SPIE, Free-Space Laser Communications X, vol. 7814, 2010.

[10] R. A. Fayadh, et al., "Establishment Network by Using FSO Link Based on MD Code for Hybrid SCM-SACOCDMA Wireless System," International Journal of Electrical and Computer Engineering (IJECE), vol. 8, no. 6, pp. 5107-5117, 2018.

[11] M. Li and M. Cvijetic, "Coherent free space optics communications over the maritime atmosphere with use of adaptive optics for beam wavefront correction,” Applied Optics, vol. 54, no. 6, pp. 1453-1462, 2015. 
[12] A. K. Majumdar, et al., "Analysis of optical communications through the random air-water interface: feasibility for under-water communications," Proceedings of SPIE, Laser Communication and Propagation through the Atmosphere and Oceans, vol. 8517, 2012.

[13] O. Korotkova, et al., "Probability density function of the intensity of a laser beam propagating in the maritime environment," Optics Express, vol. 19, no. 21, pp. 20322-20331, 2011.

[14] M. Sultana, et al., "Performance investigation of OFDM-FSO systemunder diverse weather conditions of Bangladesh," International Journal of Electrical and Computer Engineering (IJECE), vol. 8, no. 5, pp. 3722-3731, 2018.

[15] L. Cui, "Analysis of marine atmospheric turbulence effects on infrared imaging system by angle of arrival fluctuations," Infrared Physics and Technology, vol. 68, pp. 28-34, 2015.

[16] L. Cui, "Temporal power spectra over weakened marine disturbance which is examined analytically and numerically in each element," Journal of the Optical Society of America A, vol. 3, no. 9, pp. 2030-2037, 2014.

[17] Y. Baykal, "Formulation of correlations for general-type beams in atmospheric turbulence," Journal of the Optical Society of America A, vol. 23, no. 4, pp. 23889-23893, 2006.

[18] H. Gerçekcioğlu, et al., "Flat-topped Gaussian laser beam scintillation in weakly turbulent marine atmospheric medium," Optics Communications, vol. 399, pp. 24-27, 2017.

[19] M. C. Gökçe, et al., "Bit error rate analysis of MISO FSO systems," Waves in Random and Complex Media, vol. 26, no. 4, pp. 642-649, 2016.

[20] I. S. Gradshteyn and I. M. Ryzhik, "Table of Integrals, Series, and Products," Seventh Edition, Academic Press, 2007.

[21] H. Gerçekcioğlu, "Performance of annular beams in weak atmospheric turbulence for satellite laser communications," Optics Communications, vol. 439, pp. 233-238, 2019.

[22] L. C. Andrews and R. L. Phillips, "Laser Beam Propagation through Random Media," SPIE, Bellingham, Washington, 2005.

[23] S. A. Arpali, et al., "Bit error rate of a Gaussian beam propagating through biological tissue," Journal of Modern Optics, vol. 67, no. 4, pp. 340-345, 2020.

[24] Y. Baykal and H. T. Eyyuboğlu, "Scintillation index of flat-topped Gaussian beams," Applied Optics, vol. 45, no. 16, pp. 3973-3979, 2006.

[25] H. Gerçekcioğlu, "Bit error rate of focused Gaussian beams in weak oceanic turbulence," Journal of the Optical Society of America A, vol. 31, no. 9, pp. 1963-1968, 2014.

\section{BIOGRAPHIES OF AUTHORS}

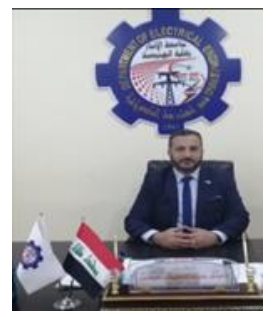

Ahmed A. Abbas is A head of Electrical Engineering Department, College of Engineering, University of Anbar from 2018 till now. He received the B.Sc. degree in Electrical Engineering/ University of Technology in 1994, he received MS.c. in communication Engineering/University of Technology in 2005 and he received $\mathrm{PhD}$ in communication Engineering/Ankara Yıldırım Beyazit University in 2017. Dr. Ahmed is researcher in optical wave propagation through random media.

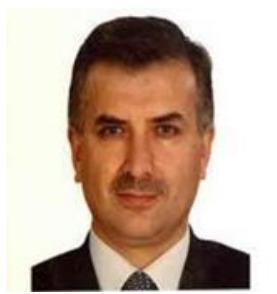

Hamza Gerçekcioğlu currently works at Republic of Turkey Ministry of Transportation and Infrastructure, Directorate General of Aeronautics and Space Technologies. Dr. Hamza does research in Engineering Education, Electronic Engineering and Communication Engineering.

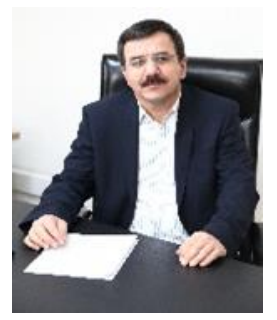

H. Haldun Göktaş received the B.Sc. degree in Electrical and Electronic Engineering/Hacettepe University in 1987. He received M.Sc. in Electrical and Electronic Engineering/Hacettepe University in 1990 and received $\mathrm{PhD}$ in Electronic Engineering/Erciyes University in 1996. Prof. Dr. Haldun is researcher in optical fiber communication and optical fiber sensors. 